\title{
Análise qualitativa mediada pelo software IRaMuTeQ: Interpretações a partir do ontem e do hoje no Sistema Único de Saúde do Brasil
}

\author{
Marcelo Prado Amaral-Rosa ${ }^{1}$ e Angela Enderle Candaten ${ }^{2}$
}

${ }^{1}$ Escola Politécnica, Programa de Pós-Graduação em Educação em Ciências e Matemática da PUCRS - Pontifícia Universidade Católica do Rio Grande do Sul, Porto Alegre/RS, Brasil | marcelo.pradorosa@gmail.com | https://orcid.org/0000-0002-3294-8141

${ }^{2}$ Escola de Medicina, Programa de Pós-Graduação em Medicina e Ciências da Saúde da PUCRS - Pontifícia Universidade Católica do Rio Grande do Sul e HCPA - Hospital de Clínicas de Porto Alegre, Porto Alegre/RS, Brasil | angela.candaten@gmail.com | https://orcid.org/0000-0002-6717-8327

Resumo: Introdução: O ponto central é o Sistema Único de Saúde do Brasil, a maior política de inclusão social da história brasileira. Debruça-se sobre as percepções de pessoas que vivenciaram a saúde brasileira antes e após a implantação do sistema. Objetivo: O objetivo foi apresentar nuances analíticas da Análise Textual Discursiva apoiada pelo software IRaMuTeQ sobre as vivências de cidadãos brasileiros acerca do ontem e do hoje do Sistema Único de Saúde. Método: É uma pesquisa de abordagem qualitativa. Os participantes são usuários da rede pública de saúde acima de 60 anos de idade $(n=39)$. O instrumento de coleta de dados foi a entrevista semiestruturada. O método para análise de dados foi a Análise Textual Discursiva, apoiado pelo software IRaMuTeQ. No software, as análises de base realizadas foram a Classificação Hierárquica Descendente e a Análise Fatorial de Correspondência. Resultados: O principal resultado foi a emersão de quatro categorias finais: i) sofrimento com a saúde pública; ii) carência associada ao antes e depois do SUS; iii) melhoria e perspectivas para o futuro do SUS; e iv) os desafios frente ao descaso com a saúde pública. Conclusões: i) para a compreensão com maior rigor frente ao processo de categorização da Análise Textual Discursiva, faz-se necessário interpretação em conjunto dos dados da Classificação Hierárquica Descendente e da Análise Fatorial de Correspondência ii) as vivências do ontem, fase que antecede a implantação do Sistema Único de Saúde brasileiro está diretamente relacionado ao sofrimento do usuário na busca por atendimento na rede pública; e iii) as vivências do hoje, são carregadas de desafios, porém, apresentam o lado positivo do Sistema Único de Saúde na vida do usuário.

Palavras-chave: Análise Qualitativa; IRaMuTeQ; Sistema Único de Saúde.

Qualitative analysis mediated by IRaMuTeQ software: Experiences of yesterday and today in the Brazilian Unified Health System

\begin{abstract}
Introduction: The central point is the Brazilian Unified Health System, the largest social inclusion policy in Brazilian history. It focuses on the perceptions of people who experienced Brazilian health before and after the implementation of the system. Objective: the aim was to present analytical nuances of the textual discursive analysis supported by the IRaMuTeQ software on the experiences of Brazilian citizens about yesterday and today of the Unified Health System. Method: it is a qualitative approach research. Participants are public health network users over 60 years of age $(n=39)$. The data collection instrument was the semi-structured interview. The method for data analysis was textual discursive analysis, supported by IRaMuTeQ software. In the software, the basic analyses performed were the descending hierarchical classification and the correspondence Factor Analysis. Results: the main result was the emergence of four final categories: i) suffering with public health; ii) deficiency associated with before and after SUS; iii) improvement and prospects for the future of SUS; and iv) challenges facing neglect with public health. Conclusions: i) the understanding, more precisely in front of the categorization process of the Analysis of Textual Discourse, it is necessary to interpreting all of the data from the Rating of the Hierarchical topdown in the Factor Analysis, correlation, ii) the experiences of yesterday, phase that precedes the implementation of the Brazilian unified health system is directly related to the suffering of the user in the search for care in the public network; and iii) the experiences of today, are loaded with challenges, however, present the positive side of the Unified Health System in the life of the user.
\end{abstract}

Keywords: Qualitative Analysis; IRaMuTeQ; Brazilian Unified Health System. 


\section{Introdução}

O ponto temático deste texto é o Sistema Único de Saúde (SUS) do Brasil. O sistema foi criado em 1988 e implementado em 1990 (Brasil, 1988; Brasil, 1990). Sustentado pelo Movimento da Reforma Sanitária Brasileira na década de 70, o SUS está inserido na Constituição Federal de 1988, na legislação ordinária e em normas técnicas e administrativas (Brasil, 1988). Com o objetivo de garantir o direito universal à saúde aos cidadãos brasileiros, tem enquanto princípios: i) a universalização do acesso às ações e serviços a todas as pessoas; ii) a equidade do atendimento considerando as necessidades específicas e desigualdades existentes em cada população; e iii) a integralidade das ações em saúde, promoção e prevenção de doenças, tratamento e reabilitação da saúde (Paim, 2018).

O SUS é considerado a maior política de inclusão social da história do Brasil (Mendes, 2013). Anteriormente, apenas os portadores da carteirinha do Instituto Nacional de Assistência Médica da Previdência Social (INAMPS) tinham direito ao acesso a uma assistência curativa, a medicina simplificada na atenção primária, sendo tratados como indigentes na atenção hospitalar (Ibid.). O SUS aboliu tal divisão e fez da saúde um direito de todos e um dever do Estado (Paim, 2018, Mendes, 2013). Isso, incorporou, mais de cinquenta milhões de brasileiros como portadores de direitos à saúde e fez desaparecer, em definitivo, o indigente sanitário (Mendes, 2013).

Logo, para a compreensão desejada sobre os aspectos da saúde pública relacionados às vivências dos usuários a respeito do SUS, recorreu-se ao tratamento de dados por meio da Análise Textual Discursiva (Moraes \& Galiazzi, 2016) apoiado pelo software de análise de dados qualitativos IRaMuTeQ (Ramos, Lima \& Amaral-Rosa, 2019). Em pesquisas qualitativas (Gray, 2012; Stake, 2011) softwares especializados estão cada vez mais presente nas análises de dados qualitativos (Gray, 2012; Stake, 2011; Mayring, 2014; Costa \& Amado, 2018).

Diante disso, declara-se a relevância e o ineditismo deste trabalho. A primeira, decorre que em 2018 o SUS completou 30 anos de sua criação (Paim, 2018) e reflexões críticas sobre as experiências das pessoas é sempre profícuo diante da importância da temática em tempos de pandemia. Já a segunda, é fruto da relação analítica entre método da Análise Textual Discursiva (Moraes \& Galiazzi, 2016) apoiada por software (Ramos, Lima \& Amaral-Rosa, 2019) na área da Saúde.

Com vistas ao direcionamento do estudo, edificou-se a seguinte questão norteadora: Quais as vivências dos cidadãos brasileiros acima de 60 anos frente à utilização do SUS no Brasil? Nesse sentido, o objetivo foi apresentar nuances analíticas da Análise Textual Discursiva apoiada pelo software IRaMuTeQ sobre as vivências de cidadãos brasileiros acerca do ontem e do hoje do Sistema Único de Saúde.

No que tange à estrutura, este artigo apresenta três seções: i) procedimentos metodológicos, na qual o ponto central são as decisões metodológicas assumidas; ii) resultados e discussões, em que o ponto negrálgico são as categorias emergentes dos dados discutidas com a literatura; e iii) conclusões, na qual se retoma a questão central e são expostas as principais representações acerca do ontem e do hoje dos usuários do SUS brasileiro.

\section{Procedimentos Metodológicos}

\subsection{Objeto e Contexto de Estudo}

O objeto do estudo são as interpretações analíticas a partir do método da Análise Textual Discursiva apoiado pelo software IRaMuTeQ (Ramos, Lima \& Amaral-Rosa, 2019), tendo por base as vivências (Carvalho et al., 2015; Mussi et al., 2007) de cidadãos brasileiros com mais de 60 anos, usuários do SUS do Brasil (Paim, 2018; Carvalho, 2013; Mendes, 2013). 
A pesquisa é enraizada na abordagem qualitativa de cunho descritivo-interpretativo (Gray, 2012; Stake, 2011, Amado, 2013), uma vez que a inteção foi captar informações sobre as vivências enquanto usuários da rede pública de saúde em diferentes momentos da vida.

O estudo é consequência de uma atividade didática da disciplina de Políticas Públicas e $o$ Contexto da Saúde Coletiva, do curso de graduação em Enfermagem, de um município da região serrana do estado do Rio Grande do Sul, no ano de 2017. A intenção foi aproximar os acadêmicos da realidade contextual que engloba a saúde pública. Logo, cada estudante, entrevistou ao menos um cidadão brasileiro com mais de 60 anos de idade, em geral membro de sua família, a respeito da utilização do SUS em diferentes momentos da vida.

Como não se configura em um trabalho decorrente de projetos de pesquisa ou fomento, $e$ sim de uma prática cotidiana de sala de aula, faz-se desnecessária aprovação pelo Comitê de Ética em Pesquisa, uma vez que as ações didáticas a serem desenvolvidas é responsabilidade do professor regente. Além disso, o foco aqui são os entendimentos provenientes das análises executadas no software-alvo e não se expõem com riscos, em nenhum momento, os participantes.

\subsection{Participantes e Corpus de Análise}

Os participantes foram usuários do SUS, acima de 60 anos de idade $(\mathrm{n}=39)$, com média de 71 anos ( $\Delta 60$ a 92 ), sendo $51 \%$ (20) do sexo masculino e $49 \%$ (19) do sexo feminino. Para a seleção dos participantes, os critérios de inclusão e exclusão foram: i) inclusão: ter 60 anos de idade (mínimo), porque assim, teriam vivências de pré e pós implantação do SUS; e ii) exclusão: em caso de profissional da área da saúde. Todos participaram de modo voluntário e consentiram com a utilização dos dados de forma acadêmica. $O$ anonimato é garantido por meio da designação da letra $P$ (Participante) com numeração arábica sequencial (P1 a P39).

A versão utilizada do software IRaMuTeQ (Ratinaud, 2014; Camargo \& Justo, 2013) é a 0.7 Alpha 2. Todas opções de entrada de dados foram mantidas de acordo com o padrão base do software (Camargo \& Justo, [s.d.]). O corpus global de análise foi constituído por 286 segmentos de texto (STs), com retenção de $82,87 \%$ (237 STs). O total de palavras do corpus foi de 10.092 , com média de 258 por participante $(\Delta 72$ a 1.310$)$. Os segmentos textuais apresentam, em média, 35 palavras, sendo 573 de ocorrência única (coeficiente Hapax $=5.68 \%$ das ocorrências).

\subsection{Instrumento de Coleta e Análise de Dados}

A coleta de dados foi realizada por meio de uma entrevista semiestruturada (Gray, 2012; Stake, 2011, Amado, 2013; Minayo \& Costa, 2018). O instrumento teve quatro perguntas abertas: i) como era a saúde no Brasil na década de 70, antes da criação do SUS?; ii) em sua percepção, como é o SUS?; iii) Após a implantação do SUS, como você avalia a saúde pública?; e iv) Em sua opinião, quais os avanços e desafios do SUS?

A entrevista foi aplicada no segundo semestre de 2017. Foram realizadas nas residências dos participantes, com média de $\sim 25$ minutos cada. Os participantes foram escolhidos conforme conveniência, sendo, via de regra, familiares ou pessoas com alto grau de proximidade dos estudantes. As informações foram sendo registradas à mão, no momento de ocorrência dos discursos, dispensando gravações de qualquer natureza. O processo analítico considera a Análise Textual Discursiva apoiado pelo software IRaMuTeQ (Ramos, Lima \& Amaral-Rosa, 2019). Fez-se a análise do corpus $(n=39)$ frente à Análise Fatorial de Correspondência (AFC) e Classificação Hierárquica Descendente (CHD) (Ratinaud, 2014; Camargo \& Justo, 2013). Enfatiza-se que, apesar do alicerce quantitativo do software, a análise ressalta a interpretação dos resultados, direcionando-se para os aprofundamentos pertinentes no que tange, em específico, ao processo de categorização do método da Análise Textual Discursiva (Moraes \& Galiazzi, 2016; Lima \& Ramos, 2017; Ramos, Lima \& Amaral-Rosa, 2019). 


\section{Resultados e Discussões}

O corpus de análise são as entrevistas dos participantes $(n=39)$. Vale lembrar que foi constituído por $\sim 10$ mil palavras (média 258 por entrevista). Destaca-se que o critério de saturação dos dados (Creswell \& Miller, 2000; Barros et al., 2017) foi atingido com base no coeficiente de Hapax (5.68\%). Quanto mais elevado o coeficiente de Hapax, maior a peculiaridade dos enunciados, portanto, considera-se o valor satisfatório (Martins et al., 2020).

No procedimento apoiado por software, as etapas da unitarização e categorização da Análise Textual Discursiva (Moraes \& Galiazzi, 2016), ocorrem de modo concomitante (Ramos, Lima \& Amaral-Rosa, 2019), com destaque para a formação de classes. Sublinha-se que as classes formadas consistem em categorias intermediárias no que se refere ao procedimento de categorização da Análise Textual Discursiva (Ibid.).

Para a obtenção dos resultados, foram executadas as análises de Análise Fatorial de Correspondência (AFC) e Classificação Hierárquica Descendente (CHD) (Camargo \& Justo, 2013; Veraszto et al., 2018). Na Análise Fatorial de Correspondência (AFC) é possível verificar as associações de dependência e independência entre cada categoria intermediária. O plano cartesiano, permite visualizar o nível de (in)dependência por meio das distâncias ou proximidades entre as variáveis (Veraszto et al., 2018). Logo, as associações de dependência, ocorrem em duas situações: i) categorias no mesmo quadrante; e ii) categorias próximas de linhas/colunas. Já as correspondências de independência, acontecem quando as categorias estão em quadrantes distintos.

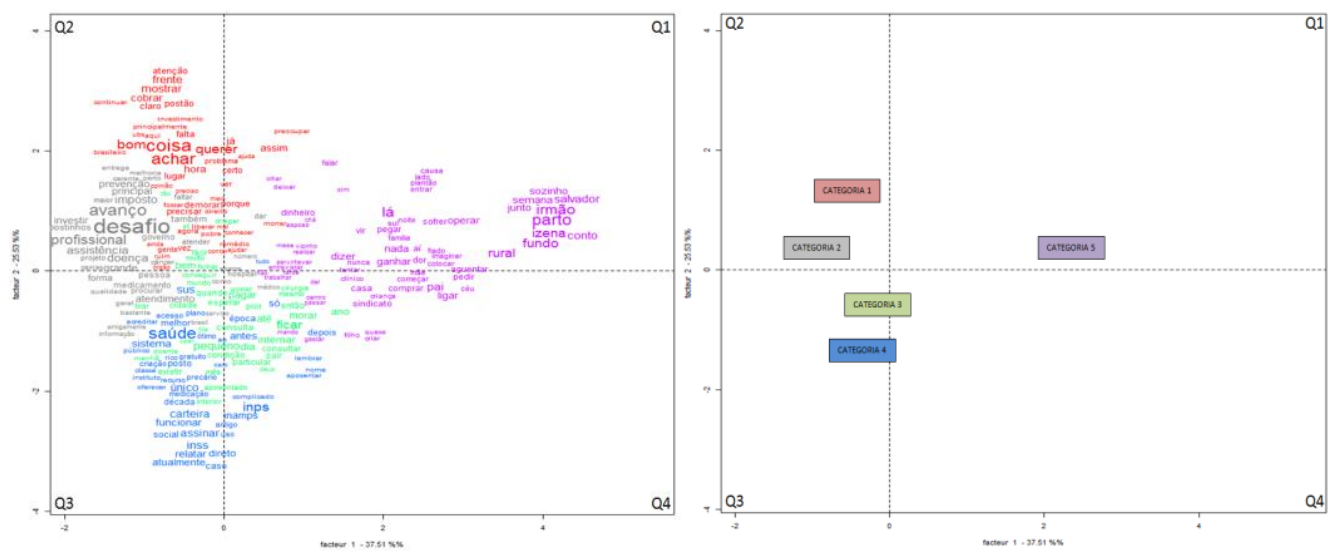

Fig. 1. AFC das palavras de cada categoria intermediária (esq.) e AFC das categorias intermediárias (dir.)

Fonte: Dados do IRaMuTeQ.

A Análise Fatorial de Correspondência (Fig.1) tem por objetivo demonstrar as possibilidades de associações entre as categorias intermediárias emergentes. O plano cartesiano da esquerda (Fig.1), corresponde à AFC das palavras que compõem cada uma das cinco categorias intermediárias. É possível perceber que as palavras variam o tamanho conforme representatividade em cada categoria, e. g., bom, achar e querer (vermelho - categoria 1), avanço, desafio e profissional (cinza - categoria 2), e saúde, INSS e INPS (azul - categoria 4). Essa representação gráfica permite a visualização do imbricamento e da aderência dos termos (Veraszto et al., 2018), favorecendo a interpretação das associações entre as categorias intermediárias.

Já no plano cartesiano direito (Fig.1), é apresentada a representação gráfica da AFC apenas das cinco categorias intermediárias formadas. Conforme a disposição espacial, há 10 formas de associações entre as categorias intermediárias, sendo oito de dependência e duas de independência, sendo possível apontar três níveis de intensidade para as associações, sendo eles: i) baixo; ii) moderado; e iii) acentuado (Quadro 1). 
Quadro 1. Associações de dependência e independência entre as categorias intermediárias.

\begin{tabular}{|c|c|c|c|c|}
\hline N.a & Conjuntos $a$ & Categorias intermediárias $a$ & Associação & $\begin{array}{l}\text { Nível-de- } \\
\text { intensidadea }\end{array}$ \\
\hline $01 a$ & la & $\begin{array}{l}\text { Categoria } \cdot 1 \cdot e \cdot \text { Categoria } \cdot 2 \mathrm{a} \\
\text { Categoria } \cdot 3 \cdot e \cdot C \text { Categoria } \cdot 4 a\end{array}$ & \multirow{7}{*}{ Dependênciaa } & Acentuado \\
\hline $03 a$ & $\| \alpha$ & Categoria $\cdot 2 \cdot e \cdot$ Categoria $\cdot 3 a$ & & Moderadoa \\
\hline 04a & \multirow{5}{*}{ III } & \multirow{5}{*}{$\begin{array}{l}\text { Categoria } \cdot 1 \cdot e \cdot \text { Categoria } \cdot 3 a \\
\text { Categoria } 1 \cdot e \cdot C \text { Categoria } \cdot 4 a \\
\text { Categoria } \cdot 2 \cdot e \cdot \text { Categoria } \cdot 4 a \\
\text { Categoria } \cdot 2 \cdot e \cdot C \text { Categoria } \cdot 5 a \\
\text { Categoria } \cdot 3 \cdot e \cdot C \text { Categoria } \cdot 5 a\end{array}$} & & \multirow{5}{*}{ Baixo } \\
\hline 05a & & & & \\
\hline $06 a$ & & & & \\
\hline $07 a$ & & & & \\
\hline $08 a$ & & & & \\
\hline $09 a$ & \multirow{2}{*}{ IVa } & Categoria $\cdot 1 \cdot e \cdot$ Categoria $\cdot 5 \mathfrak{a}$ & \multirow{2}{*}{\multicolumn{2}{|c|}{ Independênciađ }} \\
\hline 10a & & Categoria $\cdot 4 \cdot e \cdot$ Categoria $\cdot 5 a$ & & \\
\hline
\end{tabular}

Frente às interpretações para as associações entre as categorias intermediárias (Quadro 1 e Fig. 1) é possível perceber a formação de quatro conjuntos:

i) Conjunto l: o caso das associações entre as categorias 1 (As coisas boas do SUS) e 2 (Avanços e desafios do SUS), pertencentes ao quadrante 2 (Q2) no plano cartesiano; e as categorias 3 (Saúde pública era ruim) e 4 (Antes e depois do SUS), no quadrante 3 (Q3), as dependências são acentuadas, pois ambos os pares de categorias estão nos mesmos quadrantes, Q2 e Q3 respectivamente, e com elevadas proximidades;

ii) Conjunto II: o caso do pareamento entre as categorias 2 (Avanços e desafios do SUS) e 3 (Saúde pública era ruim), expõe uma dependência moderada, visto que apesar de estarem em quadrantes distintos, encontram-se em extrema proximidade no plano espacial.

iii) Conjunto III: o caso das associações entre os três pares: categorias 1 (As coisas boas do SUS) e 3 (Saúde pública era ruim); categorias 1 (As coisas boas do SUS) e 4 (Antes e depois do SUS); e 2 (Avanços e desafios do SUS) e 4 (Antes e depois do SUS), as dependências são de baixa intensidade, uma vez que as categorias estão em quadrantes distintos, próximas a linha vertical, porém com distância considerável entre si. Já o caso das associações entre a categoria 2 (Avanços e desafios do SUS) e 5 (Sofrimento com a saúde pública); e entre a categoria 3 (Saúde pública era ruim) e 5 (Sofrimento com a saúde pública), também apresentam dependência baixa entre si porque, mesmo dos quadrantes opostos e proximidade com a linha horizontal, apresentam-se muito distantes.

iv) Conjunto IV: o caso das categorias 1 (As coisas boas do SUS), 4 (Antes e depois do SUS) e 5 (Sofrimento com a saúde pública), apresenta associação de total independência. Encontram-se, cada uma em um quadrante distinto, Q2, Q3 e Q4 respectivamente, e a distância entre si é demasiada para haver algum nível de dependência.

$\mathrm{Na}$ análise de Classificação Hierárquica Descendente foi gerado um dendrograma com as cinco categorias intermediárias (Fig. 2). A classificação dos segmentos de texto (Camargo \& Justo, [s.d]) é baseada em testes estatísticos, e. g., o teste de qui-quadrado (Veraszto et al., 2018), o que permite formar categorias intermediárias na configuração mais estável (Camargo \& Justo, 2013; Veraszto et al., 2018). Na categorização indutiva, é corriqueiro a nomeação das categorias a partir dos significados comuns presentes nos discursos dos participantes que compõem cada (re)agrupamento (Moraes \& Galiazzi, 2016).

O entendimento, do universo dos usuários da rede pública de saúde é relevante já que as políticas de saúde pública desempenham papel importante nas vidas das pessoas, independente da situação social. Além disso, é valoroso compreender a construção social acerca do SUS ao longo dos anos, pelo viés do usuário, uma vez que a saúde pública é alvo constante de críticas quanto a sua eficácia, equidade e universalidade (Paim, 2018; Carvalho, 2013; Mendes, 2013). 
Assim sendo, os títulos das cinco categorias intermediárias foram gerados, a partir da interpretação dos significados dos 50 segmentos de texto de maior score ${ }^{1}$ : i) sofrimento com a saúde pública (categoria 5); ii) antes e depois do SUS (categoria 4); iii) saúde pública defasada (categoria 3); iv) as coisas boas do SUS (categoria 1); e v) avanços e desafios do SUS (categoria 2). Quanto maior o valor do score, maior a densidade e importância do ST dentro das categorias intermediárias (Ramos, Lima \& Amaral-Rosa, 2019).

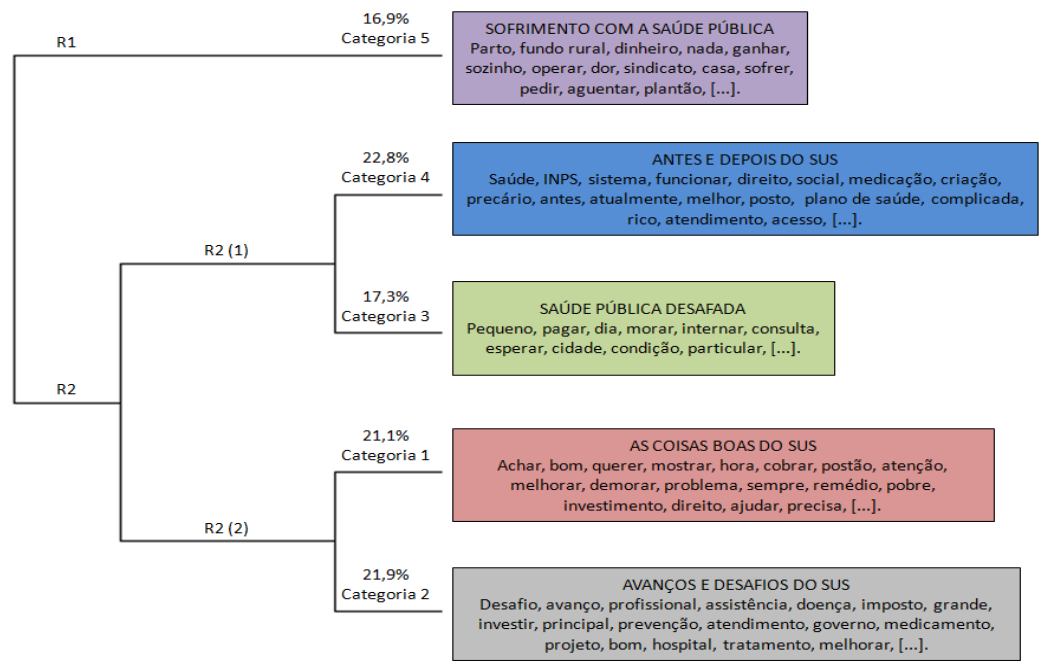

Fig. 2. Dendrograma de CHD das categorias acerca das vivências dos participantes. Fonte: Dados do IRaMuTeQ adaptados.

Constata-se na estrutura mais estável (fig. 2) (Ratinaud, 2014; Camargo \& Justo, 2013; Veraszto et al., 2018) duas ramificações: i) ramificação 1 (R1), com a categoria 5 isolada; ii) ramificação 2 (R2), subdividida em R2(1) (categorias 3 e 4) e R2(2) (categorias 1 e 2). Quanto mais próximas às categorias intermediárias, maior a afinidade contextual e os futuros (re)agrupamentos para formação das categorias finais, sob a ótica da Análise Textual Discursiva (Ramos, Lima \& Amaral-Rosa, 2019; Moraes \& Galiazzi, 2016).

A partir das interpretações baseadas nos preceitos do método da Análise Textual Discursiva (Moraes \& Galiazzi, 2016) apoiadas pelas análises no IRaMuTeQ (Ramos, Lima \& Amaral-Rosa, 2019), formou-se quatro categorias finais: i) sofrimento com a saúde pública, resultante da categoria intermediária solitária 5; ii) carência associada ao antes e depois do SUS, derivada do reagrupamento das categorias intermediárias 3 (Saúde pública defasada) e 4 (Antes e depois do SUS), na ramificação R2(1); iii) melhoria e perpectivas futuras do SUS, consequência da união das categorias 1 (As coisas boas do SUS) e 2 (Avanços e desafios do SUS), na ramificação R2(2); e iv) os desafios frente ao descaso com a saúde pública, originada das associações com níveis de intensidade relevantes entre as categorias 2 (Avanços e desafios do SUS), 3 (Saúde pública defasada) e 5 (Sofrimento com a saúde pública).

Em síntese, as formações interpretativas para cada categoria final, estão relacionadas aos focos temáticos correspondes aos discursos de seus principais representantes entre os participantes. Assim, expõem-se os principais elos formativos e os excertos de destaque, constituintes de cada uma das quatro categorias finais:

\footnotetext{
${ }^{1}$ Pontuação de score adotada: Absoluta (soma dos $\mathrm{X}^{2}$ de formas marcadas no segmento de texto). Para a inclusão das palavras em cada categoria, a associação da palavra com cada categoria é determinada pelo valor de $\mathrm{X}^{2}$ igual ou superior a 3.841 (Veraszto et al., 2018; Camargo \& Justo, [s.d.]).
} 
i) Sofrimento com a saúde pública: formou-se do isolamento dos discursos pertencentes da categoria intermediária 5 . Ao se posicionarem frente ao SUS, os participantes, discorrem sobre algumas vivências peculiares enquanto usuários da saúde pública no Brasil. Em sua maioria, são relatados momentos ocorridos antes da impantação do SUS e que carregam a mescla de dor, impotência e tristezana busca por atendimentos médicos. O excerto, a seguir, ilustra os discursos da categoria: "[...] cinco dias depois voltei para consultar e tive que pagar [...], o que tínhamos (dinheiro) foi lá para o hospital [...]" (P23, score 158.38, categoria 5).

ii) Carência associada ao antes e depois do SUS: nas categorias intermediárias 3 (Saúde pública defasada) e 4 (Antes e depois do SUS), o ponto nevrálgico das vivências dos entrevistados são os aspectos negativos relativos à saúde pública no Brasil de modo geral e do SUS. Além disso, são tecidas algumas comparações entre as condições do passado e a situação atual da saúde. As transcrições a seguir, exemplificam a construção desta categoria final: "[...] sempre com muitas filas [...]" (P35, score 167.53, categoria 4), "Digamos que mudou pouca coisa [...], o sistema era muito precário [...]" (P34, score 197.61, categoria 4), "[...] tem cidades que é bem defasada, não tem cirurgias [...]" (P9, score 40.88, categoria 3) e "[...] o SUS cobre uma massa (pessoas) muito grande e não tem estrutura, não existe o suficiente" (P26, score 39.85, categoria 3).

iii) Melhoria e perspectivas futuras do SUS: ambas categorias intermediárias que formam essa categoria final (1 e 2) estão no mesmo quadrante (Q2 - Fig.1) numa relação de proximidade, uma vez que os participantes mencionam que o SUS apresenta boas ações, porém, "[...] é claro que a gente sempre quer que melhore [...]" (P35, score 186.67, categoria 1), e entre os avanços citados estão "[...] a entrega de medicamentos de graça para a população e os tratamentos para várias doenças gratuitamente [...]" (P11, score 187.47, categoria 2), aspectos que aparecem de forma indireta relacionados aos desafios que o sistema público de saúde precisa superar para entregar um serviço de qualidade para a população.

iv) Os desafios frente ao descaso com a saúde pública: o ponto central dessa categoria final são os obstáculos do progresso do SUS frente à defasagem ao longo dos anos da saúde pública com vistas a evitar o suplício da população ao buscar por atendimento. Desse modo, as vivências dos participantes estão atreladas às diversas dificuldades enquanto usuários do sistema público de saúde, porém ao mesmo passo apresentam consciência frente a melhorias. Os excertos demonstram o cerne dos discursos que constituem a categoria final: [...] muitas pessoas sofreram, passavam fome, não tinham dinheiro e o governo não ajudava [...]" (P23, score 130.37, categoria 5), "[...] meu Deus do Céu! Tu não imaginas! Não se tinha nada, nada, nada [...]" (P26, score 37.73, categoria 5), "[...] o SUS não evolui muito, pois ainda existe muitas pessoas na fila, sem atendimento correto [...]" (P4, score 52.40, categoria 3), "O SUS veio para ajudar, mas precisa ainda de muitos ajustes para se tornar bom [...]" (P1, score 170.88, categoria 2) e "[...] agora estamos no céu! não existia médico (na saúde pública)" (P17, score 51.51, categoria 3).

Com o predomínio de uma política neoliberal, implementada pelo governo atual, a importância do SUS em tempos pandêmicos é notória e ímpar na vida dos brasileiros. Apesar da Constituição Federal assegurar o acesso à saúde como direito, vê-se, por parte dos representantes no governo brasileiro, constantes ataques com intenções de depreciar a sustentabilidade dessa política nacional e da qual boa parte dos brasileiros são dependentes (Paim, 2018). Assim, o entendimento de representações de usuários auxilia a formação de estudantes de graduação com a vivência da relevância do SUS dentro de suas casas e na sua comunidade. 


\section{Conclusões}

O propósito foi identificar as vivências de cidadãos brasileiros acima de 60 anos acerca do ontem e do hoje do SUS, com vistas ao entendimento das nuances da saúde pública tendo enquanto ferramenta analítica os preceitos da Análise Textual Discursiva apoiada pelo software IRaMuTeQ. A considerar as análises de dados, expõem-se as seguintes conclusões:

i) Frente ao procedimento de categorização: as análises textuais apoiadas pelo IRAMUTEQ permitiram identificar que o procedimento para a formação de categorias finais adequadas, que consideram os preceitos intepretativos da Análise Textual Discursiva, necessitam de análises concomitantes dos dados oriundos tanto da Análise Fatorial de Correspondência quanto Classificação Hierárquica Descendente. Verificouse que as análises isoladas não permitem a identificação por completa das associações possíveis entre as categorias intermediárias emergidas. Logo, considera-se que conclusões acerca dos procedimentos metodológicos são importantes, pois continuam a "iluminar a caixa preta" (Ramos, Lima \& Amaral-Rosa, 2019) frente às construções de dados qualitativos apoiados por softwares.

ii) Frente às vivências anteriores ao SUS: com base nas categorias intermediárias e finais, a fase anterior à implantação do Sistema Único de Saúde é um aspecto que está relacionado diretamente ao sofrimento do usuário na busca por atendimentos na rede pública. Nesse momento, os discursos trazem à tona detalhes de uma época não tão distante da saúde pública brasileira, e em tom de denúncia, evidenciam uma fase de abandono da população por parte do governo brasileiro, deixando aparente a situação da saúde nacional pré implantação do SUS.

iii) Frente às vivências do ontem e do hoje no SUS: a análise por meio das categorizações, permitiu identificar que os participantes, com base em suas múltiplas vivências enquanto usuários do SUS, acreditam ser uma ação governamental de extrema necessidade para a população e que melhorias de toda ordem são urgentes para que o serviço seja considerado satisfatório sob a ótica global de funcionamento. Ainda, constatou-se, que os discursos do ontem do SUS são mais carregados de pessimismo dentro de uma realidade mais crua e vivencial. Já, por sua vez, os discursos do hoje, apresentam o lado positivo do SUS, enunciando que apesar das evidentes melhorias urgentes, o sistema funciona com qualidade satisfatória.

\section{Agradecimentos}

CAPES - Coordenação de Aperfeiçoamento de Pessoas de Nível Superior pela concessão de bolsa de estudos de pós-doutoramento para um dos autores.

\section{Referências}

Amado, J. (2013). Manual de investigação qualitativa em educação. Imprensa da Universidade de Coimbra: Coimbra, Portugal.

Barros, N. F., Rodrigues, B. S., Teixeira, R. A. G., Oliveira, E. S. F. \& Silva, L. F. (2017). "Quantas entrevistas são suficientes?": reflexões sobre a técnica da saturação dos dados na pesquisa qualitativa. In: Oliveira, E. S. F.; Barros, N. F.; Neri de Souza, D. C. D. B. Metodologias qualitativas em diferentes cenários: saúde e educação. Goiânia: Gráfica UFG. pp. 19-33.

Brasil. (1988). Constituição da República Federativa do Brasil de 1988. Diário Oficial da União. Brasília, DF: Exército Brasileiro.

Brasil. (1990). Lei n. 8.080 de 19 de setembro de 1990. Dispõe sobre as condições para a promoção, proteção e recuperação da saúde, a organização e o funcionamento dos serviços correspondentes e dá outras providências. Diário Oficial da União. Brasília, DF: Ministério da Saúde do Brasil. 
Camargo, B. V. \& Justo, A. M. (2013). IRAMUTEQ: um software gratuito para análise de dados textuais. Temas em Psicologia, 21(2), 513-18.

Camargo, B. V. \& Justo, A. M. (S.d.). Tutorial para uso do software IRAMUTEQ. Recuperado de: https://goo.gl/22jP4X, acesso em 18-12-2018.

Carvalho, S. O. R. M., BUDÓ, M. L. D., SILVA, M. M., ALBERTI, G. F. \& SIMON, B. S. (2015). "Com pouco de cuidado a gente vai em frente": vivências de pessoas com estomia. Texto Contexto Enferm., 24(1), jan./mar., 279-87.

Carvalho, G. (2013). A saúde pública no Brasil. Estudos Avançados, 27(78), 7-26.

Creswell, J. \& Miller, D. (2000). Determining Validity in Qualitative Inquiry. Theory Into Practice, 39(3), 124-130.

Gray, D. (2012). Pesquisa no mundo real. Porto Alegre: Penso.

Lima, V. M. R., \& Ramos, M. G. (2017). Percepções de interdisciplinaridade de professores de Ciências e Matemática: um exercício de Análise Textual Discursiva. Revista Lusófona de Educação, 36, 163-177.

Martins, I. C. S., Lima, V. M. R., Amaral-Rosa, M. P., Moreira, L., \& Ramos, M. G. (2020). Handcrafted and Software-Assisted Procedures for Discursive Textual Analysis: Analytical Convergences or Divergences? In Costa, A. Reis, L. Moreira, A. (Eds). Computer Supported Qualitative Research. WCQR 2019. Advances in Intelligent Systems and Computing, vol. 1068. Springer, Cham. pp. 189-205.

Mendes, E. V. (2013). 25 anos do Sistema Único de Saúde: resultados e desafios. Estudos Avançados, 27(78), 27-34.

Minayo. M. C., \& Costa, A. P. (2018). Fundamentos teóricos das técnicas de investigação qualitativa. Revista Lusófona de Educação, 40, p. 139-153.

Moraes, R., \& Galiazzi, M. C. (2016). Análise Textual Discursiva. ljuí: Ed. Unijuí.

Mussi, F. C., Passos, L. C. S., Menezes, A., \& Caramelli, B. (2007). Entraves no acesso à atenção médica: vivências de pessoas com infarto agudo do miocárdio. Rev. Assoc. Med. Bras., 53(3), 234-9.

Paim, J. S. (2018). Sistema Único de Saúde (SUS) aos 30 anos. Ciência \& Saúde Coletiva, 23(6), 1723-1728.

Ramos, M. G., Lima, V. M. R., \& Amaral-Rosa, M. P. (2019). IRAMUTEQ Software and Discursive Textual Analysis: Interpretive Possibilities. In: Costa A., Reis L., Moreira A. (eds) Computer Supported Qualitative Research. WCQR 2018. Advances in Intelligent Systems and Computing, vol 861. Springer, Cham. pp. 58-72.

Ratinaud, P. (2014). IRAMUTEQ: Interface de $R$ pourlesAnalysesMultidimensionnelles de Textes et de Questionnaires - 0.7 alpha 2. Recuperado de: http://www.iramuteq.org.

Stake, R. E. (2011). Pesquisa qualitative: estudando como as coisas funcionam. Porto Alegre: Penso.

Veraszto, E. V., Camargo, E. P., Camargo, J. T. F., Simon, F. O., \& Miranda, N. A. (2018). Evaluation of concepts regarding the construction of scientific knowledge by the congenitally blind: an approach using the correspondence analysis method. Ciênc. Educ., 24(4), 837-857. 